\title{
Characterizing Energy Usage of a Commercially Available Ground Robot: Method and Results
}

\author{
John A. Broderick \\ Electrical Engineering: Systems, University of Michigan, Ann Arbor, Michigan 48109 \\ e-mail: jabrod@umich.edu
}

Dawn M. Tilbury

Mechanical Engineering, University of Michigan, Ann Arbor, Michigan 48109

Ella M. Atkins

Aerospace Engineering, University of Michigan, Ann Arbor, Michigan 48109

Received 20 January 2014; accepted 20 January 2014

\begin{abstract}
Energy usage by mobile robots is becoming a larger concern as autonomous systems increase mission durations. Understanding and characterizing energy usage is key to reliable mission operation. This paper presents a methodology for characterizing energy usage of a ground robot traveling at a constant speed applicable to both open and "black box" (proprietary) platforms. To address testing area limitations, we introduce a "rounded box" path to approximate straight line travel. For a case study, this methodology was applied to an iRobot Packbot on grass and asphalt surfaces. Our results show a decrease in electrical energy used with increasing speed for soft grass and constant energy usage on asphalt. For both surfaces, a similar electrical-to-mechanical efficiency curve is obtained. (C) 2014 Wiley Periodicals, Inc.
\end{abstract}

\section{INTRODUCTION}

Battery energy storage is a limiting factor for electrically powered unmanned ground vehicles (UGVs). If the battery is completely depleted, the robot is stranded and must be retrieved by a human operator, potentially in dangerous situations. To prevent battery depletion, it is necessary to estimate how much energy will be used over the remainder of the mission. Locomotion is one major energy expenditure.

Current fielded robots rely on different methods of avoiding battery depletion and UGV immobilization. The most basic, for semiautonomous or human-controlled missions, is a range estimation based on current battery state of charge and estimating energy usage based on past data [see Sadrpour, Jin, \& Ulsoy (2012) for example]. In this case, the UGV range estimate is relayed to the user, who can make decisions about aborting the mission. More autonomous systems can return to a base station to recharge [see Berenz, Tanaka, \& Suzuki (2012) for one example of scheduling charging based on a risk/reward analysis of running out of energy]. In both of these cases, an understanding of energy usage is necessary for the methods to function.

Here, we focus on evaluating currently fielded robots. There exist a number of commercially available robots (such as the Packbot and Talon) and many more research platforms at laboratories throughout the world. Many commercial platforms are in operation in military, police, and search-and-rescue operations. Based on our discussion with our research collaborators in the U.S. Army, these energy characterizations are needed to maximize the performance of robots that are currently in the field.

In this paper, we present a methodology for determining energy usage by a small ground robot, and we apply this methodology to the Packbot as a case study. This methodology is based on empirical energy data recorded during UGV operation, not determined from a terramechanics model of the system. While this methodology is general, it is particularly helpful for characterizing and understanding commercially available robots, where internal configurations are unknown due to proprietary constraints ("black box"). This methodology characterizes energy usage by determining the following information: 1) energy usage per distance traveled at a given speed, 2) the most efficient speed on a given terrain, and 3) a terrain-independent electrical-tomechanical efficiency curve. From these data, a path planner can optimize the path based on the known terrains and mission goals to reduce energy consumption. Additionally, this methodology can be used to compare different robots for a given application or aid in designing a robot to meet certain mission goals. Our methodology consists of conducting a series of tests at a constant forward velocity; to create a sufficiently long path, we introduce a "rounded box" path. Preliminary results were presented in Broderick, Tilbury, and Atkins (2012).

This methodology is not based on a knowledge of the internal configuration of the robot. The methodology only requires a way to drive the robot on a specified path at a constant velocity and record the electrical and mechanical

Journal of Field Robotics 31(3), 441-454 (2014) (c) 2014 Wiley Periodicals, Inc.

View this article online at wileyonlinelibrary.com • DOI: 10.1002/rob.21507 
energy used during the test. In the case of the Packbot presented in this work, we did not have access to the internal details, due to the proprietary black box nature of the robot, and we could only calculate the mechanical energy to within a scaling factor.

This paper is organized as follows. Section 2 summarizes relevant research. Section 3 presents the proposed methodology for characterizing the energy usage of a ground robot at a constant speed. Section 4 describes the Packbot platform used as a case study for this methodology. Results from the tests on the Packbot are presented in Section 5, and the conclusions are presented in Section 6.

\section{BACKGROUND}

Ground robot energy usage has been studied previously in a variety of settings. Richmond, Mason, Coutermarsh, Pusey, \& Moore (2009) present an overview of recent work, focusing on locomotion and power. A case study comparing energy usage and coverage was presented by $\mathrm{Mei}, \mathrm{Lu}, \mathrm{Hu}$, and Lee (2004). They consider an omnidirectional robot covering an open area with no obstacles and using three different coverage schemes: scan lines, spiral, and square spiral. Simulating the robot at different velocities, they calculated the efficiency of coverage paths. By comparing efficiencies at different velocities, the most efficient velocity is determined for the particular robot. Mei, Lu, Hu, \& Lee (2005) also present an experimental movement power model of a ground robot at different velocities. This work adds to their earlier power analysis by looking at the other primary battery limitation: total energy storage. Boice et al. (2010) present a comparison of power used by the Packbot while traveling over different terrains at different speeds. Using motor current measurements, they conclude that traveling faster draws more current, but no analysis is done for energy use over a distance.

Power use requirements for tracked vehicles is given in Morales et al. (2006) and Morales, Martinez, Mandow, Garcia-Cerezo, and Pedraza (2009), including losses due to track slip caused by turning. The power required to drive the vehicle at a constant velocity is modeled as linear in the absolute velocity of track speed. This research models energy use over a distance, not the power draw at each point in time. In Morales et al. (2009), the authors present some experimental results that show similar trends with decreasing total energy usage at higher velocities, but their paths are much shorter and focus on analyzing energy lost due to turning.

An in-depth, well-to-wheel analysis of an electric vehicle is presented in Campanari, Manzolini, and de la Iglesia (2009). A similar analysis can be carried out for small tracked ground vehicles such as the Packbot; however, such an analysis is beyond the scope of this research and, in our case with the Packbot, is not possible due to the black box nature of the robot. We are interested in a simple, empir- ical calculation of energy usage that can be used for path planning or range estimation.

For automotive applications, fuel consumption at different speeds has been studied and modeled [see El-Shawarby, Ahn, \& Rakha (2005), Rakha, Ahn, Moran, Saerens, \& den Bulck (2011), and Wang, Fu, Zhou, \& Li (2008)]. Since fuel is the main energy source for the vehicles, measuring fuel consumption is analogous to the energy usage calculations in this paper. In all three papers, a minimum fuel consumption per distance traveled is found in the $60-80 \mathrm{~km} / \mathrm{h}$ range, depending on specific vehicle configurations. Since there is a large difference in scale between the vehicles studied in those papers and the ground robots studied in this paper, it is necessary to repeat the analysis for ground robots. In particular, aerodynamic forces become very large at high speeds for automobiles [see Wong (2008)]; for small ground vehicles, the low speeds and low profile result in low aerodynamic drag.

\subsection{Energy-usage Application}

Estimation of ground robot range based on battery energy is highly dependent on being able to predict future energy needs. Saha, Goebel, Poll, \& Christophersen (2007) present work on estimating range based on historical data and anticipated operation conditions. Sadrpour et al. (2012) present an algorithm to estimate the energy required to complete the mission based on knowledge of future mission goals and terrain. Ceraolo and Pede (2001) estimate the remaining range for an electric car based on battery dynamics and estimated future current draw. The results presented in this paper can be used to augment these algorithms to better estimate future energy usage based on a knowledge of desired future velocities and terrains to be traversed. Zhang, Wang, Kobayashi, \& Shirai (2012) present a range calculation for electric vehicles based on nine different criteria, including current location, battery state of charge, and driving style. Data from this research (or similar tests for different robots) can be used to augment range estimation methods.

For autonomous robots, Berenz et al. (2012) present a method for determining when to return to a recharging station. They use a risk/reward analysis to determine the best time to return to the recharging station. One critical part of the algorithm is estimating the remaining range, which can be characterized using the methods described in this paper.

In addition to estimating stored energy availability in batteries, the energy consumption of a ground robot can be used to help plan future paths based on energy usage. Ooi and Schindelhauer (2009) derive a path planner for a mobile communication network based on minimizing energy consumption. This method can be augmented with the results presented here for better prediction of energy costs. Similar methods can be used to better optimize other path planners, 
consistent with ideas presented in Barili, A., Ceresa, M., and Parisi (1995).

\subsection{Track-ground Interaction}

The theory of terramechanics dates back to the initial work by Bekker (1969). These principles have been extended by Wong to form the basic foundation of terramechanics (Wong, 2008). For a detailed analysis of many aspects of skid steering and tracked vehicles, see Wong and Chiang (2001). From an empirical perspective, we are concerned with two main aspects: tractive effort and rolling resistance.

The tractive effort is the force that the track applies to the ground. For tractive effort, the friction coefficient is based on slip between the track and the ground (Wong, 2008). While tractive effort and slip are necessary components of understanding UGV mobility, we are interested in energy analysis. To estimate the tractive force in our tests, we would need to experimentally determine the slip-friction curve and develop a slip estimator to use during the tests. Research in slip detection is ongoing and not a focus of this paper.

Rolling resistance, another focus of terramechanics, is one source of mechanical energy loss for ground vehicles. Track-ground (and wheel-ground) interactions have been well studied in the literature. Wong presents the basic theory of track-ground interaction (Wong, 2008). For both wheels and tracks, one of the primary sources of energy loss is through compaction of the terrain. The pressure required to compact the soil is key in the derivation. Wong treats this pressure solely as a function of the depth of the track (wheel) sinkage.

Grahn extended the analysis for wheel-ground interaction by including a dependence for the pressure on compaction velocity (Grahn, 1991). The author found that the rolling resistance decreased as the wheel velocity increased from 0 to $16.6 \mathrm{~km} / \mathrm{h}$. Pope found a $9.3 \%$ decrease in rolling resistance as the wheel velocity increased from 0.036 to $0.274 \mathrm{~km} / \mathrm{h}$ (Pope, 1971). Crenshaw investigated the rolling resistance in high-speed tests and found three regions of interest: decrease in rut depth and rolling resistance with increasing velocity at low $(0-37 \mathrm{~km} / \mathrm{h})$ speeds, rut depth and resistance increases with velocity at medium $(37-93 \mathrm{~km} / \mathrm{h})$ speeds, and decreases at high speeds (above $93 \mathrm{~km} / \mathrm{h}$ ) (Crenshaw, 1971). Shmulevich summarized the previous results and created a simulation model to predict the effect of velocity on rigid-wheel performance for off-road terrain (Shmulevich, Mussel, \& Wolf, 1998). Their model showed a decrease in the motion resistance ratio for driven, rigid wheels. While these authors focused on wheel-ground interactions, we could not find any extensions of these models to tracked vehicles. We expect similar trends to hold for tracked vehicles.

In addition to energy loss due to compaction, tracked vehicles encounter energy loss due to skid steering. Guo and Peng (2013) present results for energy loss due to steering as a function of turning radius. Using a power loss model for turning with tracked robots, the authors show that at smaller turning radii, there is larger power loss and one track switches from propulsion to braking. Choosing a sufficiently large turning radius limits the difference in torques between the motors and results in little power loss compared to straight line travel. While their tests were performed on sand, we would expect similar results for the terrains used in this research. Their work justifies our use of a rounded box test path with a sufficiently large turning radius to approximate straight-line travel.

\section{TESTING METHODOLOGY}

As ground robots become more widespread, there is a much greater need for methods and standards to analyze robot performance. In this paper, we are interested in one particular aspect of robot performance: energy usage while moving. Previous standards have been focused on robot speed (ASTM Standard E2829, 2011), common terrains (ASTM Standard E2826, 2011), and obstacles (ASTM Standard E2801, 2011). While these standards are beneficial, they do not address energy usage. Our method adds the metric energy used for motion.

Energy usage for straight-line motion and turning must both be characterized. The test methodology presented in this paper is general, but our tests focus on straight-line energy usage on consistent, traversable terrain. One example of predominantly straight-line motion, and the motivation behind our work, is an area coverage task. The coverage task consists of long, straight segments connected by short turning segments. The energy usage is a function of many variables; this methodology considers speed and terrain as the primary variables affecting energy use.

The methodology for determining energy usage consists of selecting a series of tests, recording the necessary data during the tests, and processing the data to analyze energy usage after the tests. Figure 1 depicts the important aspects of our method. First, a set of test paths and trajectories is designed based on the desired velocities and terrains for which energy use will be characterized; wide turns can be introduced to the planned paths based on area constraints. Using an autonomous or semiautonomous controller, the $\mathrm{UGV}$ is driven based on the computed trajectories. If necessary, the robot can be augmented with additional sensors, e.g., GPS for localization, to complete the feedback controller. During the tests, the electrical voltage and currents, motor speeds, and distance traveled are recorded from the UGV. Additional sensors, such as GPS or inertial measurements, may also be recorded and used by an autonomous driving controller to follow the reference trajectory for each test. After all the tests are run, the recorded data are analyzed to produce the following characterizations: 


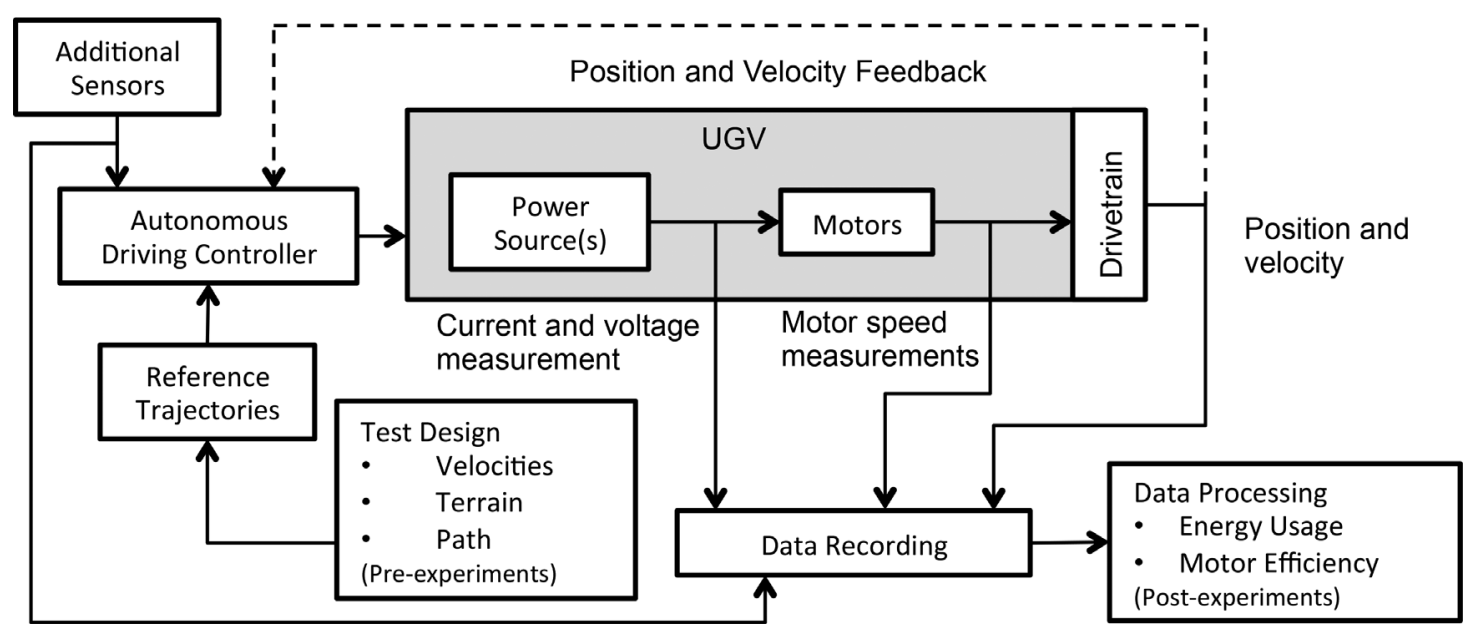

Figure 1. Flow of methodology.

1. Energy usage per distance traveled for a specific terrain

2. Most efficient speed for straight-line travel for a specific terrain

3. Terrain-independent electrical-to-mechanical efficiency curve

As the metric, we chose energy usage per distance traveled, instead of instantaneous power usage, to analyze longduration missions for ground robots. As mission duration increases, the total energy stored becomes more important than instantaneous power draws since energy used per distance shows the most efficient way to travel between different points. While power can be integrated to measure energy usage, measuring energy usage per distance changes the metric from a time-based measurement to a distancebased measurement.

To characterize ground robot energy usage, we propose a series of tests, each with the robot traveling at a constant speed. Ideally, these tests would be run in a straight line to avoid any additional energy loss due to turning. Because of limited operating areas, a "rounded box" path can be used. This path consists of straight sides with sufficiently large radius turns between the sides. The sides of the box should be as long as possible given operating area constraints. To obtain sufficient data, the total length of the path should be a significant portion of the vehicle's range.

Turns should have a radius sufficiently larger than the robot track or wheel base to minimize the amount of lateral slip at the contact points. Using a power consumption model for turning, such as the one presented in Guo and Peng (2013), the minimum radius that meets a desired power tolerance (i.e., less than $10 \%$ increase in power draw due to turning) can be computed. While the radius will depend on terrain and robot characteristics, we found that a turn radius of about eight times the track length was sufficient for our setup; we expect other robots to be similar.
For each of the independent variables, namely speed and terrain, a set of desired test points must be defined. For speed, a maximum and minimum speed should be chosen based on robot capabilities and the desired application. Intermediate speeds can be chosen based on the fineness of the data desired; more tests produce more data points at the cost of additional resources required for testing. Five to eight test speeds will provide a good tradeoff between quantity of data recorded and testing duration requirements for ground robot applications in which test time is constrained.

Terrain selection for the tests should be based on the required application. While there will always be variations in terrain parameters in real-world applications, a set of basic terrains can be chosen to predict the general performance of the robot. This set should include at least one nondeformable terrain (e.g., asphalt) and one deformable terrain (e.g., grass or sand) for a general robot designed for outdoor off-road missions.

The ground robot should be driven at the desired speed. This can be achieved through purely autonomous operation or semiautonomous operation, with the speed regulated by the controller and a human steering the robot. Autonomous operation requires more setup and sensors, but it provides more repeatability between tests. If the robot already has autonomous capabilities, this entails programming the robot to follow the path at the desired speed. Otherwise, an additional controller, either onboard the robot or on an external computer, must be designed to drive the robot on the prescribed trajectory. If necessary, a localization sensor, e.g., GPS, can be added to complete the autonomous feedback. In both cases, the controller must be well-designed to limit overshoot in steering and maintain the reference trajectory in the presence of measurement noise. For semiautonomous operation, a user interface must be provided that allows for selection of the speed. 
There are two main ways of measuring energy usage: change in energy storage (e.g., battery state of charge) or integrating instantaneous power measurements over time. The battery or other power source must provide up-to-date energy storage, while the instantaneous battery power can be calculated from the battery voltage and current measurements, if a direct battery power measurement is not available. Instantaneous mechanical power can be calculated from the motor speed and torque, which is proportional to motor currents.

After the set of tests is complete, the recorded data are analyzed to compute the energy per distance traveled and the efficiency of the electrical to mechanical conversion, which includes the motor and additional power component efficiencies. While the total average energy per distance for the entire trajectory is a useful metric, breaking the tests up into shorter segments enables extraction of more information about the statistics of energy usage. The length of the shorter segments should be based on the quality of the sensors used: tests with higher-quality sensors can be broken into shorter segments while still limiting sensor noise impact on results. The electrical-to-mechanical efficiency is calculated by dividing the mechanical energy output by the electrical energy input, and it represents losses due to the motor, power electronics, and other drivetrain components.

Alternatively, the cost of transport, defined as the energy used per meter per kilogram, can be calculated by dividing the energy per distance by the platform mass. This metric is commonly used to assess and compare bipedal robots [for example, see Collins, Ruina, Tedrake, \& Wisse (2005)]. While this makes the total energy used dependent on the current system configuration, it also allows for estimating energy usage when the UGV has an increased mass due to additional loads (e.g., sensors, manipulators, etc.).

This methodology computes an energy characterization for straight-line travel of a ground robot. It does not attempt to study complex ground-robot interactions, but it creates an empirically determined energy characterization of driving. For complex and variable terrains, these tests can serve as a basis for estimating energy usage by determining a scale factor as the robot operates. We leave a derivation of such methods for future work.

As the field of ground robots continues to develop, a standard set of proving tests can be created to compare different robots in the different settings, similar to specifications used in the automotive industry [SAE International (2012), for example]. These standard tests can be devised through robotic competitions, as described by Jacoff et al. (2012).

\section{CASE STUDY: PACKBOT PLATFORM AND TEST SETUP}

For our tests, we used an iRobot Packbot, shown in Figure 2. The University of Michigan Mobile Robotics Lab (UM MRL)

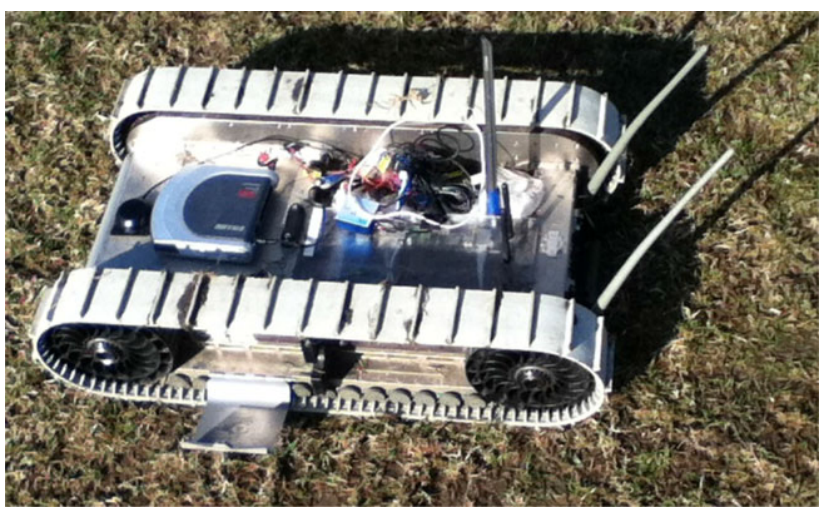

Figure 2. Packbot used in experiments.

provides an external interface to the proprietary Packbot interface. The UM MRL interface accepts forward velocity $v$ and angular velocity $\omega$ commands and provides data feedback from the Packbot. This interface is run on a Gumstix processor attached to the Packbot, and all communication takes place over a wireless network using a standard router located on the robot. Both devices are powered by the Packbot battery and consume less power than the drive system when active.

The UM MRL interface also provides real-time data from the Packbot, including the electrical and mechanical energy usage information discussed in the previous section. In this paper, we use two different energy values: electrical energy input and mechanical energy output. The mechanical power is calculated from the motor current, which is proportional to motor torque and the motor shaft speed. Since the motor speeds are not provided by the interface, they can be calculated from the ground robot forward and angular velocities, as calculated by the interface from the wheel encoders, using the Packbot width $2 B$ and track drive wheel radius $R$. The UM MRL interface thus provides

$$
\begin{aligned}
P_{\text {mech }} & =K_{m} K_{r}\left(I_{l} \omega_{l}+I_{r} \omega_{r}\right) \\
& =K_{m} K_{r}\left(I_{l} \frac{v-\omega B}{R}+I_{r} \frac{v+\omega B}{R}\right),
\end{aligned}
$$

where $K_{m}$ is the motor constant and $K_{r}$ is the gear ratio from the motors to the drive wheels. Due to proprietary data restrictions, $K_{m}$ and $K_{r}$ are unknown values. We therefore utilize the scaled mechanical power $P_{\text {mech }} /\left(K_{m} K_{r}\right)=$ $I_{l} \omega_{l}+I_{r} \omega_{r}$ in this paper. Since we are dealing with a black box model of the robot, we do not know if the motor current measurements occur directly before the motors or if there are additional electronic components between the sensor and the motors. Therefore, we treat motor and any additional electronics together as a unit. This may result in inaccurate mechanical power calculations from Eq. (1); however, we will assume that any errors are small in the range in which the motors are being operated. 


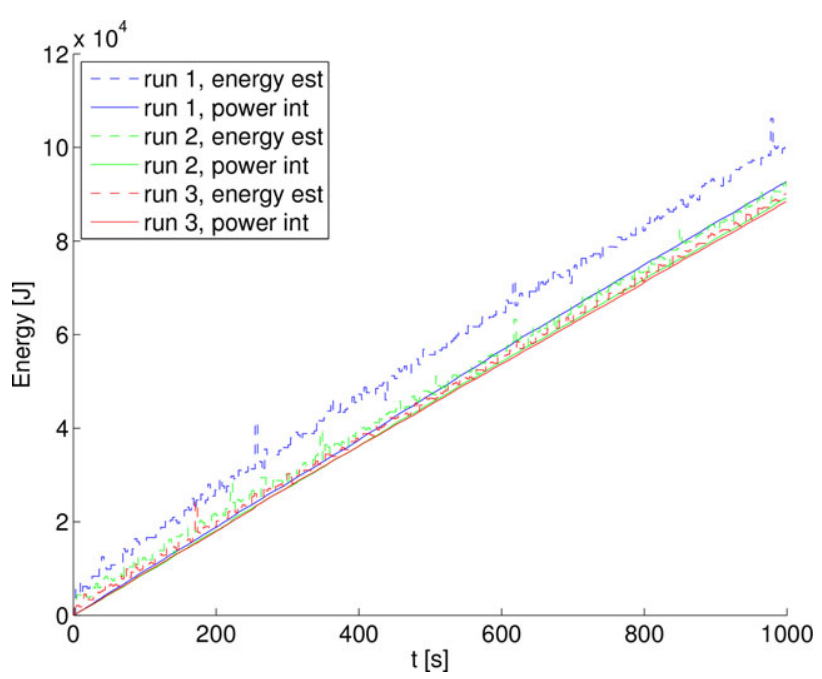

Figure 3. Integrating battery power produces comparable results to the battery energy estimate.

To filter the motor current data, we used Tikhonov regularization to reduce spikes in the measured current (Tikhonov and Arsenin, 1977). We imposed smoothness constraints on the current values to limit current spikes in the recorded data. Regularization minimized the following equation:

$$
\left|I_{r, l}-I_{\mathrm{op}} w_{r, l}\right|^{2}+\lambda^{2}\left|L w_{r, l}\right|^{2},
$$

where $w_{r, l}$ is the first-order difference in the respective measured currents, $L$ is the differential operator, $I_{\mathrm{op}}$ is the integral operator, and $\lambda$ is the regularization parameter. Each motor current was smoothed individually. We selected $\lambda=1$ to reduce motor current spikes without flattening the data significantly. This method was chosen over a simple low pass filter to limit the amount of data lost due to filter transients.

The Packbot data allow two methods to measure electrical energy usage: 1) estimation of remaining battery life and 2) the integral of the instantaneous battery power draw. For our setup, the Packbot estimates the battery energy remaining, presumably based on the state of charge of the battery, and the battery draw. We record the battery draw and integrate over time to produce our own estimate of power used. Figure 3 compares these two energy calculations for three consecutive experiments on the same battery charge. As described in detail later, these trials consist of the Packbot running at a constant speed for a certain distance. The energy used for the second and third trials is nearly identical and the first run ends with a similar slope between the two energy estimates. The discrepancy in the first trial is presumed to be caused by overestimating the energy remaining when the battery is mostly full. Similar trends are present in other sets of consecutive experiments.

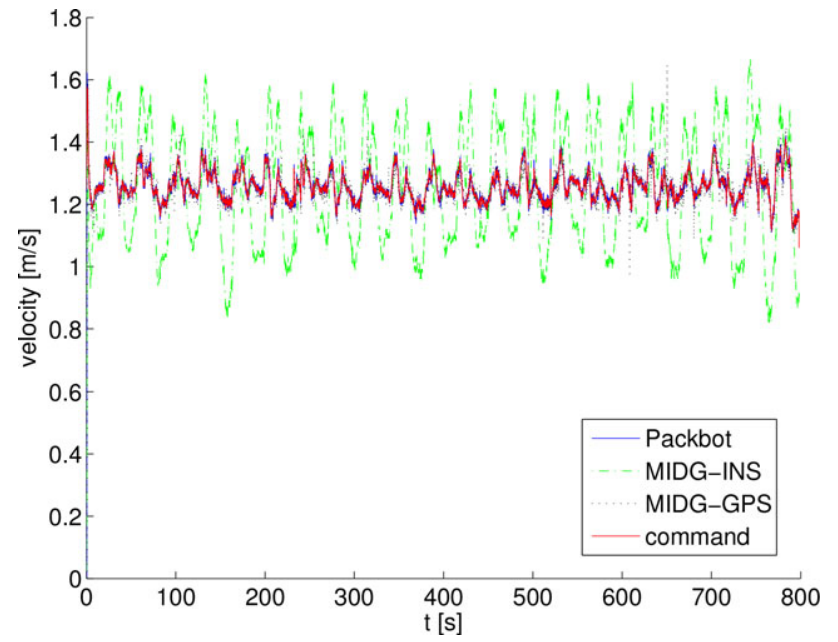

Figure 4. Comparing forward velocities from Packbot and MIDG, $v=1.25 \mathrm{~m} / \mathrm{s}$.

Also note that the battery energy estimate fluctuates up and down over the course of the trial. Because of these observations, we chose to use the integral of the battery power as our method to calculate the electrical energy used by the Packbot.

Additionally, the battery power draw includes power for peripheral electronics. To measure the auxiliary power, we recorded the power data while the Packbot was stationary with all electronics operating for about $6,000 \mathrm{~s}$. The average power usage over test was $30.8 \mathrm{~W}$, with the maximum and minimum measurements 32.1 and $30.1 \mathrm{~W}$, respectively. To calculate the energy used for motion, we subtracted the average power for peripheral devices from the battery data field before integrating.

To examine differences due to current battery state, we ran each test several times on the same battery charge. The tests were run on flat terrain. Figure 4 shows the velocity profile for one test on asphalt using three different velocity data fields recorded during the tests: track encoders (Packbot), Global Positioning System (GPS), and Inertial Navigation System (INS) values. The periodic variations are due to the slight slope in the surface used for testing.

For localization, the Packbot was augmented with a MIDG IIc INS (Microrobotics, Inc., 2011), which includes a GPS sensor. The MIDG provides $50 \mathrm{~Hz}$ localization data from an extended Kalman filter, integrating GPS, accelerometer, gyro, and magnetometer data to provide threedimensional location and attitude information. Both the raw GPS and filtered INS outputs were recorded. We ultimately turned off the magnetometer input to the Kalman filter due to interference from the Packbot, after which the heading was estimated from GPS and the integral of angular velocity about the vertical $z$ axis (turn rate). An additional Gumstix processor interfaces with the MIDG and relays the data 


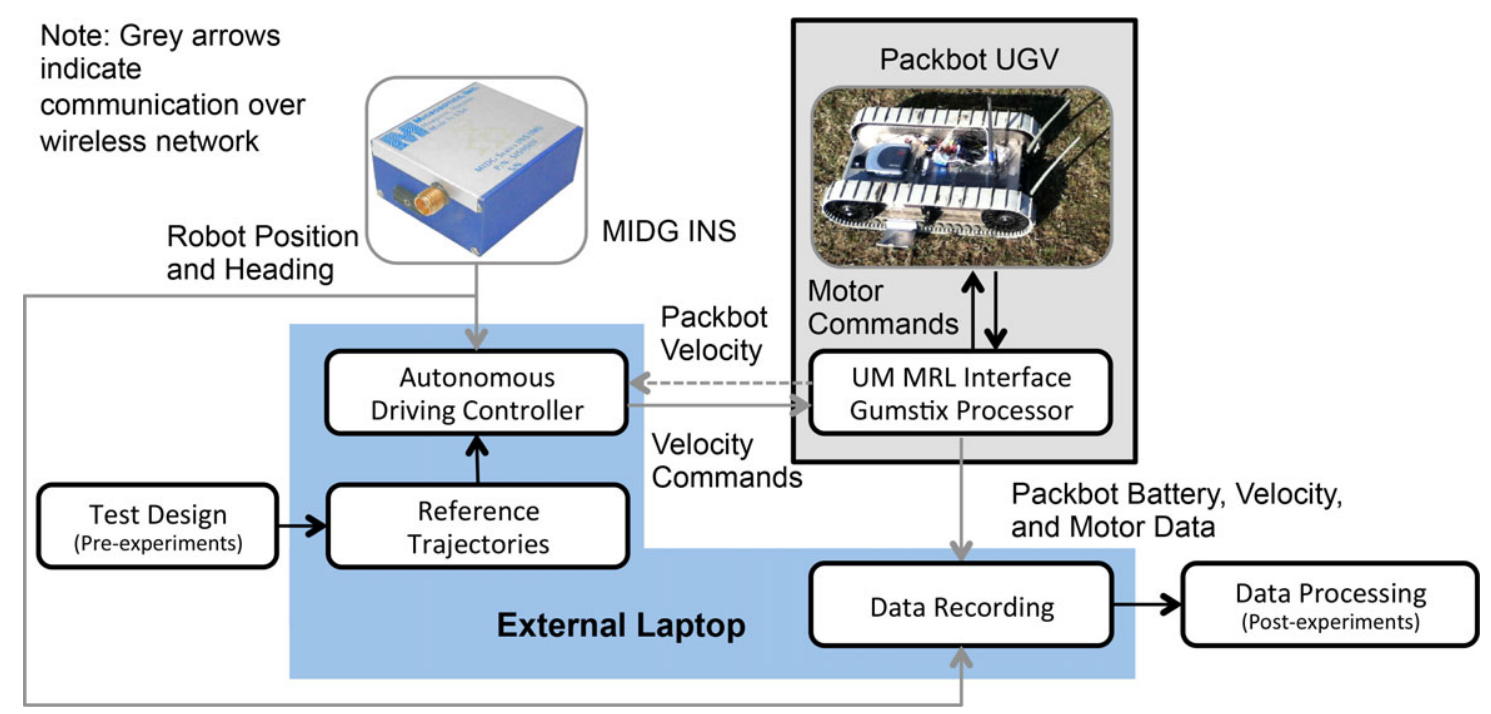

Figure 5. Schematic of data flow for the Packbot setup.

over the wireless network. The MIDG and its electronics are powered by an external battery.

To drive the Packbot along a reference trajectory, we used the following nonlinear trajectory-following controller to calculate the commanded forward velocity $v_{c}$ and turn rate $\omega_{c}$ (Kanayama, Kimura, Miyazaki, \& Noguchi, 1990):

$$
\left[\begin{array}{c}
v_{c} \\
\omega_{c}
\end{array}\right]=\left[\begin{array}{c}
v_{r} \cos \theta_{e}+K_{x} x_{e} \\
\omega_{r}+v_{r}\left(K_{y} y_{e}+K_{\theta} \sin \theta_{e}\right)
\end{array}\right] .
$$

In this controller, $v_{r}$ and $\omega_{r}$ are the reference forward velocity and angular velocity, $\theta_{e}$ is the heading error, $x_{e}$ is the longitudinal position error, $y_{e}$ is the lateral position error, and $K_{x}, K_{y}$, and $K_{\theta}$ are control gains. This controller is proven to be stable using a Lyapunov analysis (Kanayama et al., 1990). For these experiments, we use the position $(x, y)_{\text {INS }}$ and heading $\theta$ for position control.

Figure 5 shows the different subsystems active for our test setup. This figure expands the online portion of Figure 1 to show the details of the test setup. The controller and data recorder are implemented on an external laptop, accepting data over a wireless network from the MIDG and the UM MRL interface. With data being acquired by two different systems, the data must be synchronized. Table I summarizes all the recorded data fields. Driving commands are calculated based on a reference trajectory and position updates from the MIDG, and they are transmitted over the wireless network.

This controller assumes that the robot can immediately respond to new commands, though in reality there are communication and acceleration delays. Figure 6 shows the Packbot following an L-shaped trajectory of moving forward $5 \mathrm{~m}$, turning 90 degrees, then moving another $5 \mathrm{~m}$ forward. The dashed lines represent the reference trajectory.

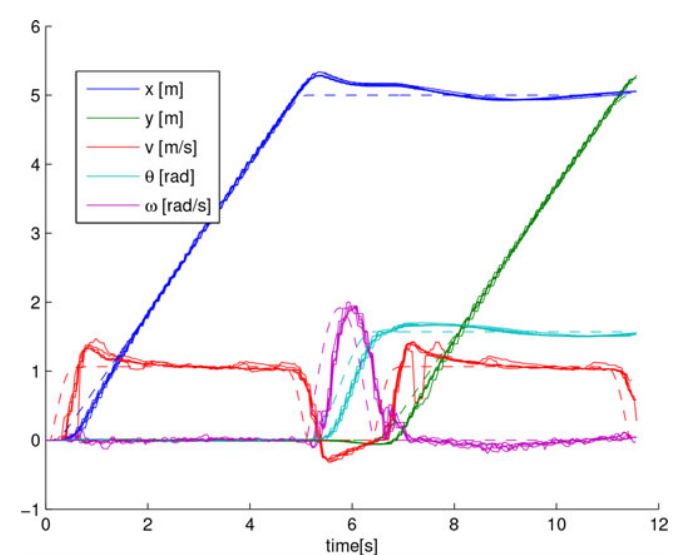

Figure 6. Minimal delays in Packbot while completing an L-shaped trajectory.

The actual trajectories from five different runs are shown in the figure and consistently have a 0.3 s delay in responding to commands. Notice that the delay also causes overshoot on the velocity as the robot tries to return to the desired location. We decided that this delay was acceptable for our application.

We executed the rounded box paths on grass and asphalt surfaces to compare the energy use on different surface types. The asphalt presents a hard surface that does not exhibit sinkage and provides good traction for the Packbot. The grass allows for more slippage and sinkage. The initial grass tests, denoted as "soft grass," occurred in the winter on a sports field.

The rounded box path was $35 \mathrm{~m}$ long and $15 \mathrm{~m}$ wide with a corner radius of $6 \mathrm{~m}$. The size of the box was based on limitations of the area under consideration and our test 


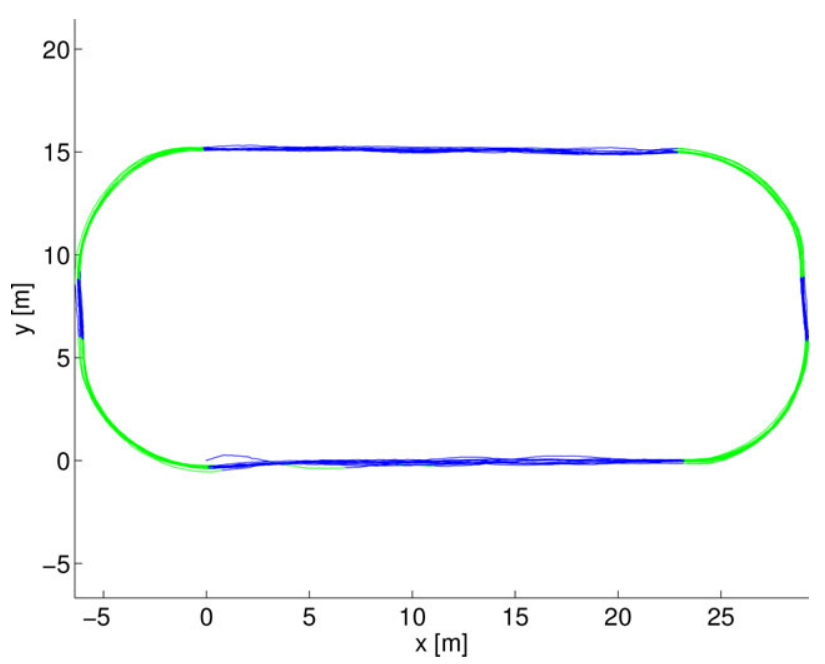

Figure 7. Rounded box trajectory, divided into straight and turn segments based on commanded turn rate, $v=1.25 \mathrm{~m} / \mathrm{s}$, on asphalt.

setup. Based on experiments presented by Guo and Peng (2013), as discussed in Section 2.2, we determined that the $6 \mathrm{~m}$ corner radius would not introduce significant energy loss due to turning. For our robot, this radius is 8.6 times the track length and 15 times the separation between the tracks.

For the Packbot, the total length of the path for our tests was chosen to be $1,000 \mathrm{~m}$, approximately one quarter of the Packbot's range. Figure 7 shows the executed path of 11 laps around the rounded box.

The tests were run at at the following speeds: $0.75,1.0$, $1.25,1.5$, and $1.75 \mathrm{~m} / \mathrm{s}$. The Packbot used in these experiments had a top speed of approximately $2 \mathrm{~m} / \mathrm{s}$. We decided to limit our tests to $1.75 \mathrm{~m} / \mathrm{s}$ to allow the Packbot to drive faster than the nominal speed if needed to maintain the planned trajectory. We did not go lower than $0.75 \mathrm{~m} / \mathrm{s}$ because we were interested in looking at speeds that would be practical while covering large fields; slower speeds would mean prohibitively large energy losses due to constant electrical loads.

Additionally, we had access to a grass runway at a local airport. We were able to run the Packbot straight along the length of the runway, as well as running the "rounded box" configuration at the same location to compare energy usage between straight and turn trajectories, verifying the assumption that the turns do not introduce significant energy loss. These tests are labeled as "hard grass" since they were run in the summer when the ground was hard. The length of the runway is $1.04 \mathrm{~km}$, though not all runs were for the full length of the runway. For simplicity in setting up the straight line tests, we used a constant speed controller with human input for steering to keep the Packbot on the runway. Table II summarizes the tests conducted to explore Packbot energy usage.
Table I. Data recorded.

\begin{tabular}{lccc}
\hline Measurement & Source & Variable & Units \\
\hline velocity & Packbot & $v$ & $\mathrm{~m} / \mathrm{s}$ \\
turn rate & Packbot & $\omega$ & $\mathrm{rad} / \mathrm{s}$ \\
battery power draw & Packbot & $P_{\text {batt }}$ & $\mathrm{W}$ \\
battery energy remaining & Packbot & $E_{\text {batt }}$ & $\mathrm{J}$ \\
motor currents & Packbot & $I_{r, l}$ & $\mathrm{~A}$ \\
position (GPS and INS) & MIDG & $(x, y)_{\text {GPS,INS }}$ & $\mathrm{m}$ \\
heading & MIDG & $\theta$ & degrees \\
velocity (GPS and INS) & MIDG & $\left(v_{x}, v_{y}\right)_{\mathrm{GPS}, \mathrm{INS}}$ & $\mathrm{m} / \mathrm{s}$ \\
\hline
\end{tabular}

Table II. Summary of tests run.

\begin{tabular}{lcc}
\hline Surface & Path & Speeds $(\mathrm{m} / \mathrm{s})$ \\
\hline Asphalt & Rounded box & $0.75,1,1.25,1.5,1.75$ \\
Hard grass & Rounded box & $0.75,1.75$ \\
Hard grass & Straight line & $0.75,1.75$ \\
Soft grass & Rounded box & $0.75,1,1.25,1.5,1.75$ \\
\hline
\end{tabular}

\section{CASE STUDY: TEST RESULTS AND ANALYSIS}

Here, we present the data acquired following the methodology described in Section 3. Specifically, we present the energy usage per distance traveled for a set of speeds on two different terrains, and the terrain-independent electrical-tomechanical efficiency curve.

Figure 8 shows the electrical and scaled mechanical power usage for a portion of a test on asphalt, with turn and straight segments delineated in different colors. We used the turn rate command to differentiate between the different segments, using a threshold value of $\omega_{\text {thresh }}=$ $0.05 \mathrm{rad} / \mathrm{s}$. The resulting $x-y$ plot is shown in Figure 7. While it is difficult to see from the graphs, the turn segments do have a higher average power use. Table III summarizes the average power used at different speeds and surfaces for the straight and turn segments. Based on these data, we conclude that the turns do introduce extra power draw, although the increase is less than $7 \%$. The periodic variation visible in Figure 8 is due to a slight slope of the surface where the test was conducted.

\subsection{Energy usage}

For each run, we recorded the energy used, as described in Section 4 , and divided by the actual distance traveled, as recorded by the GPS. Each test was divided into individual laps around the rounded box trajectory based on the GPS position. Figure 9 shows the cost of transport for the different tests. As described in Section 3, the electrical energy per distance traveled is divided by the mass of the Packbot, in this case $20 \mathrm{~kg}$, to produce the cost of transport. The boxes show the spread of the electrical energy used 


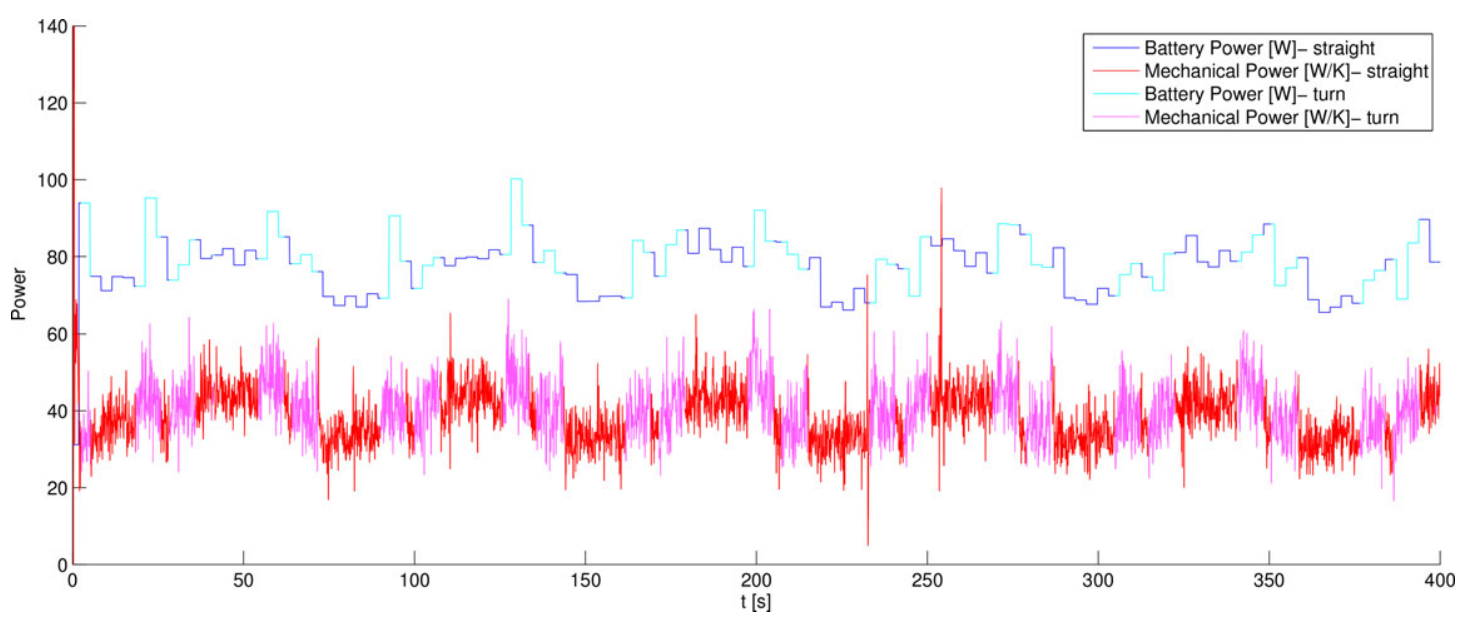

Figure 8. Electrical and mechanical power over time for one run, $v=1.25 \mathrm{~m} / \mathrm{s}$ on asphalt.

Table III. Average battery power draw for different speeds.

\begin{tabular}{|c|c|c|c|c|c|}
\hline Speed & $0.75 \mathrm{~m} / \mathrm{s}$ & $1 \mathrm{~m} / \mathrm{s}$ & $1.25 \mathrm{~m} / \mathrm{s}$ & $1.5 \mathrm{~m} / \mathrm{s}$ & $1.75 \mathrm{~m} / \mathrm{s}$ \\
\hline \multicolumn{6}{|c|}{ (a) Soft grass } \\
\hline Straight & $76.9 \mathrm{~W}$ & $87.1 \mathrm{~W}$ & $102 \mathrm{~W}$ & $115 \mathrm{~W}$ & $127 \mathrm{~W}$ \\
\hline Turn & $82.3 \mathrm{~W}$ & $93.6 \mathrm{~W}$ & $104 \mathrm{~W}$ & $117 \mathrm{~W}$ & $126 \mathrm{~W}$ \\
\hline \multicolumn{6}{|c|}{ (b) Asphalt } \\
\hline Straight & $62.1 \mathrm{~W}$ & $70.7 \mathrm{~W}$ & $76.2 \mathrm{~W}$ & $91.4 \mathrm{~W}$ & $98.2 \mathrm{~W}$ \\
\hline Turn & $64.9 \mathrm{~W}$ & $74.8 \mathrm{~W}$ & $79.5 \mathrm{~W}$ & $95.7 \mathrm{~W}$ & $101 \mathrm{~W}$ \\
\hline
\end{tabular}

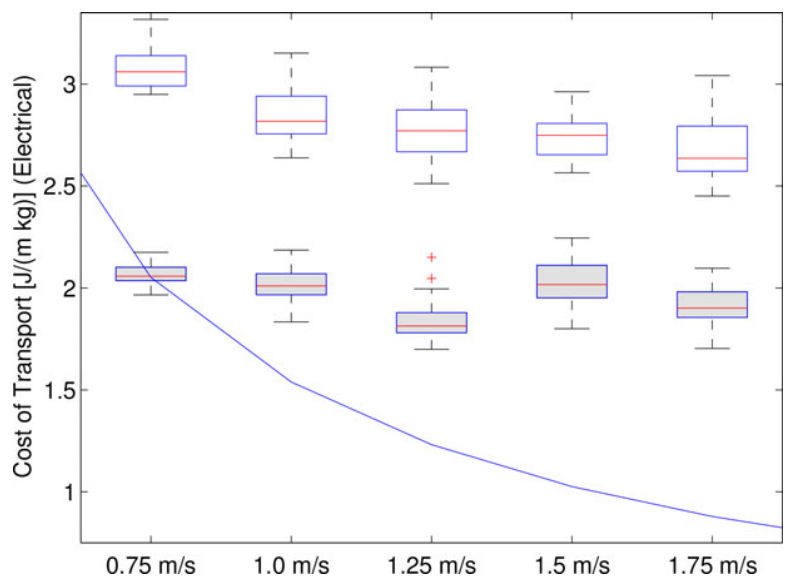

Figure 9. Statistical analysis of electrical cost of transport for Packbot (white, soft grass; gray, asphalt) compared with peripheral electronics (blue line).

for locomotion; the red center lines show the median value, the boxes show the $25 \%-75 \%$ percentile of the data, and the whiskers show the limit of the data not considered as outliers, with outliers individually plotted. The continuous blue line shows the energy used per meter traveled of the
Table IV. Variance and mean for individual laps $(\mathrm{J} / \mathrm{m})$.

$$
0.75 \mathrm{~m} / \mathrm{s} \quad 1.0 \mathrm{~m} / \mathrm{s} \quad 1.25 \mathrm{~m} / \mathrm{s} \quad 1.5 \mathrm{~m} / \mathrm{s} \quad 1.75 \mathrm{~m} / \mathrm{s}
$$

(a) Soft grass

\begin{tabular}{|c|c|c|c|c|c|}
\hline Mean & 61.45 & 57.09 & 55.45 & 54.88 & 53.47 \\
\hline $\begin{array}{l}\text { Variance } \\
\text { (b) Asphalt }\end{array}$ & 3.85 & 6.90 & 5.90 & 3.50 & 9.05 \\
\hline Mean & 41.36 & 40.24 & 36.74 & 40.61 & 38.14 \\
\hline Variance & 1.20 & 2.23 & 2.93 & 4.19 & 3.68 \\
\hline
\end{tabular}

Table V. Percentage decrease in energy usage from $0.75 \mathrm{~m} / \mathrm{s}$ in average energy per meter.

\begin{tabular}{lcccc}
\hline & $1.0 \mathrm{~m} / \mathrm{s}$ & $1.25 \mathrm{~m} / \mathrm{s}$ & $1.5 \mathrm{~m} / \mathrm{s}$ & $1.75 \mathrm{~m} / \mathrm{s}$ \\
\hline Soft grass & $7.09 \%$ & $9.76 \%$ & $10.69 \%$ & $12.99 \%$ \\
\hline
\end{tabular}

peripheral electronics. The power draw is assumed to be constant for different speeds; dividing the power draw by the speed gives the desired value of energy per meter.

There are two main trends in the data. The tests on grass show that the average cost of transport decreases as speed increases. Table $\mathrm{V}$ lists the percentage decrease in energy usage for the different velocities tests, showing up to $12.99 \%$ decrease in energy usage for the fastest speed. For the asphalt cases, the cost of transport is significantly lower and is roughly constant over the speeds tested. Looking at Figure 9, we can compare the distribution of energy usage from the individual segments. On the soft grass, there is considerable overlap in the distributions, with the median value (red line) decreasing with increasing speed. The asphalt tests show a decreased variation in energy used, presumably due to the more uniform, harder surface. Table IV shows the sample variance of mean for the 


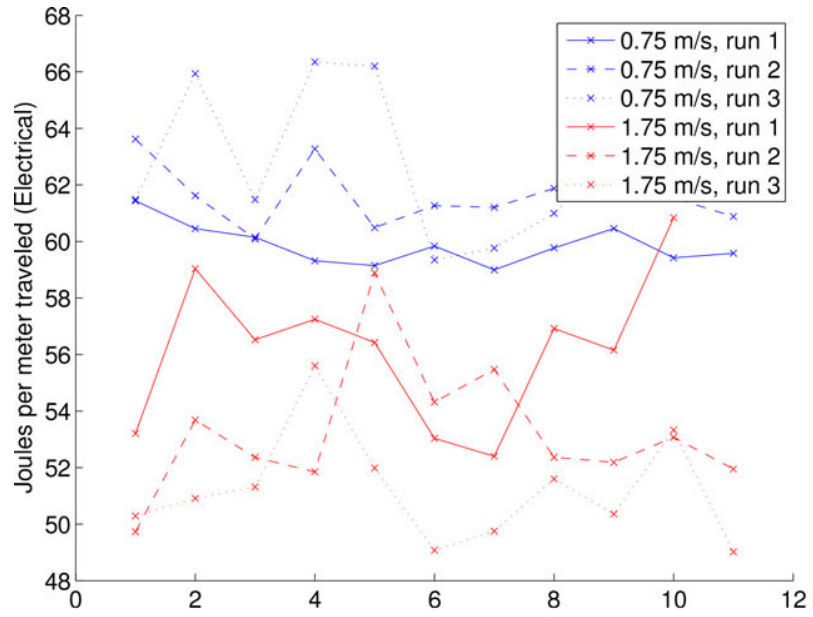

(a) Soft grass, laps

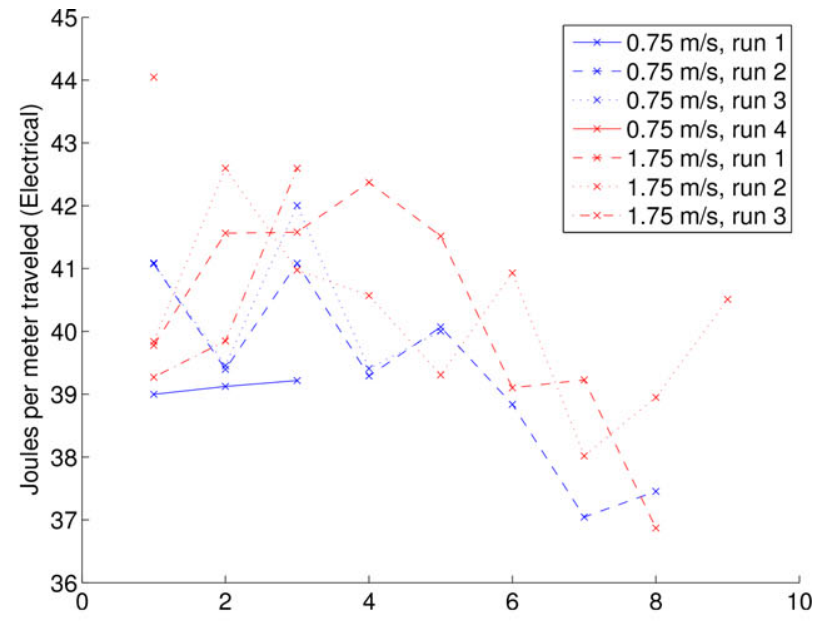

(b) Hard grass, $100 \mathrm{~m}$ segments

Figure 10. Energy usage for consecutive $100 \mathrm{~m}$ segments.

Table VI. Energy per meter traveled of straight line and rounded box tests on hard grass $(\mathrm{J} / \mathrm{m})$.

\begin{tabular}{lcc}
\hline & $0.75 \mathrm{~m} / \mathrm{s}$ & $1.75 \mathrm{~m} / \mathrm{s}$ \\
\hline Straight line (hard grass) & 39.46 & 40.60 \\
Rounded box (hard grass) & 42.02 & 44.45 \\
Rounded box (asphalt) & 41.36 & 38.14 \\
\hline
\end{tabular}

different trajectories. Using the statistical variance, we see that the variance is smaller for the runs on asphalt, with the exception of $1.5 \mathrm{~m} / \mathrm{s}$. The variance can be influenced by many factors, including differences in terrain between runs.

Looking at consecutive laps around the rounded box, we see considerable variation in the energy use required. Figure 10(a) shows the electrical energy usage of subsequent laps for 0.75 and $1.75 \mathrm{~m} / \mathrm{s}$. Figure 10(b) shows the straight line tests broken into $100 \mathrm{~m}$ segments. These tests show less variability than the soft grass tests.

Comparing the energy used for peripheral electronics with the energy used for motion shows that, on asphalt, for $0.75 \mathrm{~m} / \mathrm{s}$, the energy usage is split approximately equally between locomotion and peripheral electronics. For $1.75 \mathrm{~m} / \mathrm{s}$ on asphalt, the energy used for peripherals is about half the energy used for locomotion.

To compare the energy consumption differences between straight line travel and the rounded box trajectory, the results of the tests on hard grass are shown in Table VI. For $0.75 \mathrm{~m} / \mathrm{s}$, the rounded box tests used $6.3 \%$ more energy than the average of the straight traversals down the airport runway and $9.6 \%$ more for $1.75 \mathrm{~m} / \mathrm{s}$. These tests show that the rounded box is a good estimate for straight line travel on the hard grass. These values are similar to the

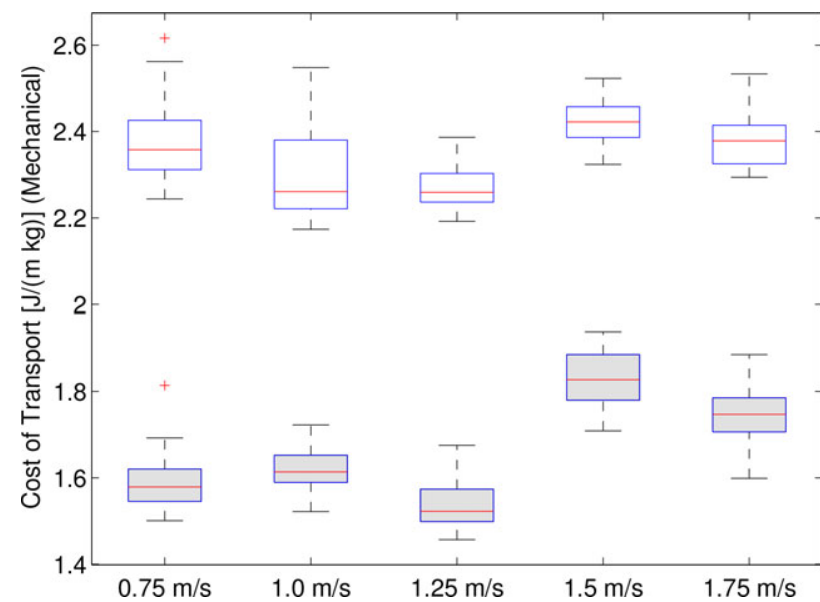

Figure 11. Scaled mechanical cost of transport for Packbot in different conditions (white, soft grass; gray, asphalt).

values obtained for the asphalt tests, rather than the softer grass, showing a strong dependence on soil conditions in the energy required for travel.

In addition to the electrical energy usage, we looked at the mechanical energy used. Figure 11 shows the scaled mechanical cost of transport for the same tests presented in Figure 9. The trends present in the electrical cost of transport are not present in these results; the tests on soft grass stayed relatively constant while the energy usage for the asphalt tests generally increased with speed.

Since the mechanical energy used is closely related to the motion resistance (assuming a constant normal load), we expected to see the same trends summarized in Section 2.2 for soft grass; however, the energy used does not 
Table VII. Scaled mechanical energy per meter traveled of straight line and rounded box tests on hard grass $\left[\mathrm{J} /\left(\mathrm{K}_{\mathrm{m}} \mathrm{K}_{\mathrm{r}} \mathrm{m}\right)\right]$.

\begin{tabular}{lcc}
\hline & $0.75 \mathrm{~m} / \mathrm{s}$ & $1.75 \mathrm{~m} / \mathrm{s}$ \\
\hline Straight line (hard grass) & 31.09 & 38.52 \\
Rounded box (hard grass) & 31.91 & 42.34 \\
Rounded box (asphalt) & 31.76 & 34.93 \\
\hline
\end{tabular}

decrease with speed. There are many possibilities for this discrepancy; however, due to the use of a fielded robot and not a dedicated test setup, we are unable to conclusively analyze the track-ground interactions of the Packbot during use. We leave for future work further experimental analysis of the track-ground interactions for the Packbot.

On both asphalt and the hard grass surfaces, the scaled mechanical energy use increases with speed. In particular, Table VII shows the comparison of the straight line and rounded box trajectories for hard grass. In both cases, the energy usage increases, although the increase is greater for the rounded box trajectory. For the hard grass scenario, this implies that the rounded box does increase the resistance, though it is not the only factor involved.

\subsection{Electrical-to-Mechanical Efficiency}

Power loss in the motors and drive train is one source of loss in the system. Using the electrical and scaled mechanical energy, discussed in the previous section, we can calculate the efficiency of the conversion from electrical to mechanical energy. Since we are dealing with a "black box" model of the Packbot, we must make the assumption that the gear ratio from the track drive wheels to the motors is constant. Based on this assumption, we can parametrize the efficiency based on the track speeds.

To compute efficiency, we define

$$
\begin{gathered}
\text { efficiency }=\eta=\frac{\text { Power Out }}{\text { Power In }}=\frac{K_{m}\left(I_{l} \omega_{l}+I_{r} \omega_{r}\right)}{\text { Battery Power }} \\
=\frac{K_{m} K_{r}\left(I_{l} \frac{v-\omega B}{R}+I_{r} \frac{v+\omega B}{R}\right)}{\text { Battery Power }} .
\end{gathered}
$$

Since $K_{m}$ and $K_{r}$ are unknown, we calculate the scaled efficiency, $\eta /\left(K_{m} K_{r}\right)$. In addition to regularizing the data, we excluded some data points where, due to experimental conditions, the battery power input dropped down to near 0 , resulting in large efficiency values that could skew the final results.

Figure 12 shows the scaled efficiencies from the different tests. Efficiency varies with vehicle speed, and the efficiency values are consistent at the same speed on different runs, including on different surfaces. This figure also corresponds closely to measured efficiency curves for ground robots [see Logan, Pentzer, Brennan, \& Reichard (2012) for an example]. Also, interestingly, scaled efficiency for the rounded box trajectory on hard grass at $1.75 \mathrm{~m} / \mathrm{s}$ is significantly higher than that for other surfaces, possibly due to the low state of battery charge for that test. Since dc motor efficiency varies with speed and torque [Say and Taylor (1986), Chap. 10], it is interesting to note that there is little difference in efficiency between surfaces. Since higher speeds and lower torques are more efficient, we conclude that the speed increase is more important for efficiency than the increase in output torque.

Since our tests approach the maximum speed of our Packbot, we could not test higher speeds to see if the efficiency continues to increase or where the peak efficiency occurs. In fact, as described in Logan et al. (2012), one method for motor selection and gear design is to put the maximum efficiency of the system at the largest power output. We expect the efficiency curve to have a peak, and we assume this peak to be around $2 \mathrm{~m} / \mathrm{s}$, the maximum speed of the robot, since we only observed efficiency increasing in the range of tested speeds. Above this speed, the efficiency decreases until the motor stall torque is reached and the motor outputs no work. To shape the portion of the curve that we could not test, we added three extra data points at higher speeds: 0.9 at $2.5 \mathrm{~m} / \mathrm{s}, 0.8$ at $3.5 \mathrm{~m} / \mathrm{s}$, and 0.7 at $4.5 \mathrm{~m} / \mathrm{s}$. Without including these points, the polynomial fit increased rapidly above $1.75 \mathrm{~m} / \mathrm{s}$, an infeasible result. We used a fourth-order polynomial fit, which resulted in the following equation:

$$
\begin{aligned}
\text { scaled efficiency }= & \frac{\eta}{K_{m} K_{r}}=0.0055 v^{4}-0.033 v^{3}-0.036 v^{2} \\
& +0.3586 v+0.5613
\end{aligned}
$$

This curve partially explains the results presented earlier; the tests at a higher speed are operating in a more efficient regime of operation and have lower power loss in

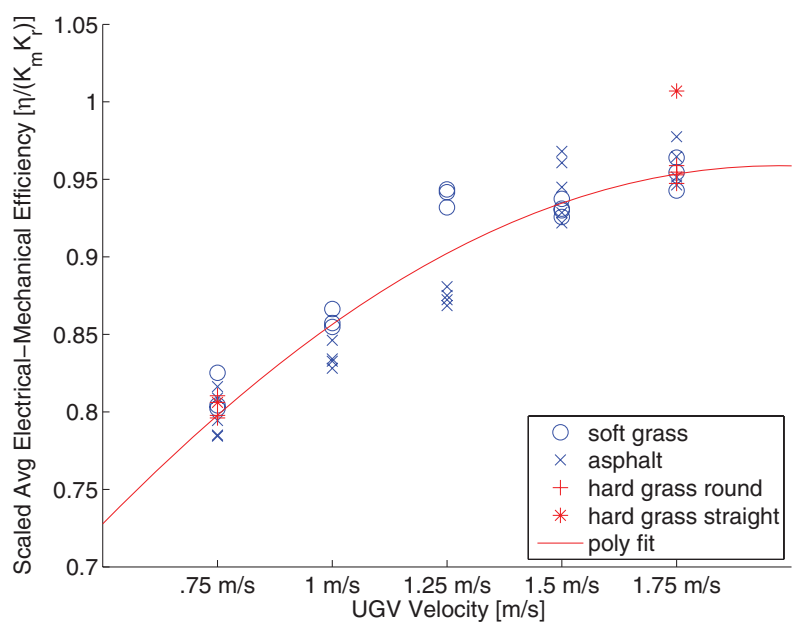

Figure 12. Efficiency values consistent over the different runs. 
Table VIII. Estimated Packbot range at constant speed from full battery.

\begin{tabular}{lccccc}
\hline & & & & & \\
& $0.75 \mathrm{~m} / \mathrm{s}$ & $1 \mathrm{~m} / \mathrm{s}$ & $1.25 \mathrm{~m} / \mathrm{s}$ & $1.5 \mathrm{~m} / \mathrm{s}$ & $1.75 \mathrm{~m} / \mathrm{s}$ \\
\hline Grass & $3.39 \pm 0.06 \mathrm{~km}$ & $3.94 \pm 0.12 \mathrm{~km}$ & $4.33 \pm 0.13 \mathrm{~km}$ & $4.60 \pm 0.11 \mathrm{~km}$ & $4.90 \pm 0.11 \mathrm{~km}$ \\
Asphalt & $4.21 \pm 0.06 \mathrm{~km}$ & $4.88 \pm 0.10 \mathrm{~km}$ & $5.66 \pm 0.16 \mathrm{~km}$ & $5.68 \pm 0.19 \mathrm{~km}$ & $6.22 \pm 0.19 \mathrm{~km}$ \\
\hline
\end{tabular}

Table IX. Total distance and percentage of battery depleted for the tests.

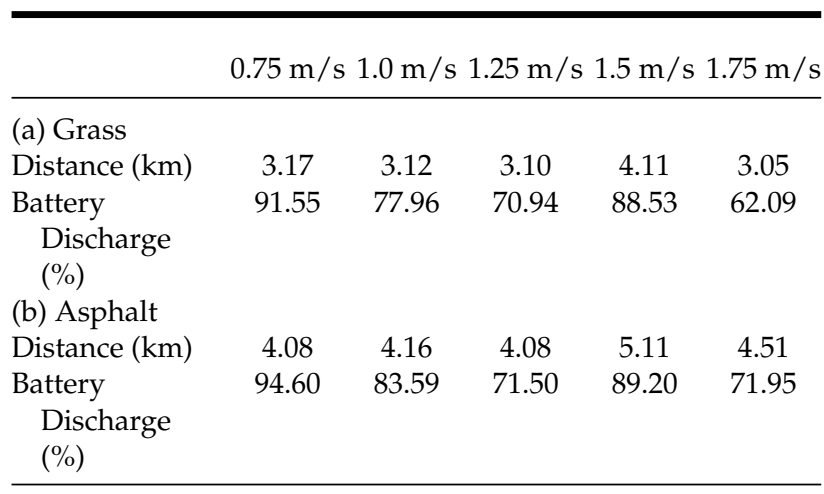

the motors and drivetrain. Path planners can use this curve to plan efficient operation.

\subsection{Range Prediction}

Using the methodology proposed in Section 3, we have characterized energy usage for the Packbot. As one example of usage of the data, we present simple range estimates based on the traveling speed. In this case, we will assume a constant speed for the entire duration of the mission.

Using the data from Figure 9 and taking into account the average power for peripherals, we can estimate the range of the Packbot traveling at a constant forward velocity $v$ using the following formula:

$$
\text { range }=\frac{E_{b a t t}}{E_{\text {dist }}+P_{p e r} v} \frac{(J)}{(J / m)},
$$

where $E_{\text {batt }}$ is the available battery energy at the start of the traversal (J), $E_{\text {dist }}$ is the average energy expended per unit distance from Table IV $(\mathrm{J} / \mathrm{m})$, and $P_{\text {per }}$ is the average power for peripherals (W). Table VIII lists the estimated range for a fully charged battery for the Packbot, with error values based on the variance of energy usage. The accuracy of these estimates depends on many factors, including the variability of the terrain, the accuracy of battery charge estimate, and the variance of the energy usage data. Table IX shows the range from the tests presented in the previous section along with the percentage of the battery used. All the tests for a particular velocity were run on the same bat-
Table X. Comparing $E_{\text {dist }}$ and $P_{\text {per }} / v$.

\begin{tabular}{lccc}
\hline$v$ & $E_{\text {dist }}$ (soft grass) & $E_{\text {dist }}$ (asphalt) & $P_{\text {per }} / v$ \\
\hline $0.75 \mathrm{~m} / \mathrm{s}$ & 61.45 & 41.36 & 41.04 \\
$1.25 \mathrm{~m} / \mathrm{s}$ & 55.45 & 36.74 & 24.62 \\
$1.75 \mathrm{~m} / \mathrm{s}$ & 53.47 & 38.14 & 17.59 \\
\hline
\end{tabular}

tery with minimal breaks between tests, although the tests were not run until the battery was depleted.

One interesting aspect of the error values in Table VIII is the increase of the error range as speed increases, even though the variance of the energy usage data varies with speed. This is due to the relative size of $P_{\text {per }} / v$ and $E_{\text {dist }}$. Table $\mathrm{X}$ compares the values of $E_{\text {dist }}$ and $P_{\text {per }} / v$ from our tests. When $v$ is larger, the load from electronics is less per unit distance, so the variance of $E_{\text {dist }}$ has a larger effect. In addition, modifying the setup to change $P_{\text {per }}$ would not result in a uniform effect for all speeds; slower speeds would have a larger range increase when $P_{\text {per }}$ decreases than faster speeds.

This range estimate can be used to augment battery range estimation algorithms to improve accuracy. This is a simple range estimation; research into estimating range based on robot history and estimated future action is ongoing (Sadrpour et al., 2012). Battery models under load can also affect the range and thus the results of these projections [see Lin et al. (2013) for an example of a battery model].

\section{CONCLUSIONS}

We have presented a methodology for characterizing energy usage for a mobile ground robot and results obtained by applying this methodology to the iRobot Packbot. This methodology entails running the robot through a series of tests at different speeds and on different terrains. From these tests, we record the energy used per distance traveled and the electrical-to-mechanical efficiency. These data can be used to produce more efficient path plans and to better estimate the remaining range.

For the Packbot, our methodology showed that the robot required less energy when traveling on asphalt than on soft grass. On the soft grass, the energy requirements decreased with increasing speed, while the same trend was not present on asphalt. The electrical-to-mechanical efficiency 
increased with speed up to the maximum speed allowed by the Packbot, but it did not depend on the terrain.

In the case of our particular coverage application, we had assumed that the UGV would be more efficient at lower speeds, creating a need for balanced tradeoffs. However, after completing this characterization of energy usage for the Packbot, we discovered that there were more complex relationships between speed and efficiency and adjusted our trajectory planning based on these results, including the electrical-to-mechanical efficiencies in the planning process [see Broderick, Tilbury, and Atkins (2014)].

Data from these experiments can be used to improve battery life prediction, and to better inform autonomous planning and operation protocols for small robotic vehicles. If the energy per distance at different speeds is known for the terrains to be traveled upon, a range prediction can be generated. Alternatively, an energy efficiency curve can be used in conjunction with a more detailed terrain interaction model to estimate the range for a given battery state of charge. Models for energy usage with more complicated maneuvers or more variable terrain are left for future research.

\section{ACKNOWLEDGMENTS}

This research was supported in part by the Automotive Research Center (ARC) at the University of Michigan, with funding from government contract DoD-DoA W56H2V-042-0001 through the U.S. Army Tank Automotive Research, Development, and Engineering Center.

Disclaimer: Reference herein to any specific commercial company, product, process, or service by trade name, trademark, manufacturer, or otherwise, does not necessarily constitute or imply an endorsement, recommendation, or favoring by the U.S. Government or the Department of the Army (DoA). The opinions of the authors expressed herein do not necessarily state or reflect those of the U.S. Government or the DoA, and shall not be used for advertizing or product endorsement purposes.

UNCLASSIFIED: Distribution Statement A. Approved for public release.

\section{REFERENCES}

ASTM Standard E2801 (2011). Standard test method for evaluating emergency response robot capabilities: Mobility: Confined area obstacles: Gap.

ASTM Standard E2826 (2011). Standard test method for evaluating emergency response robot capabilities: Mobility: Confined area terrains: Continuous pitch/roll ramps.

ASTM Standard E2829 (2011). Standard test method for evaluating emergency response robot capabilities: Mobility: Maneuvering tasks: Sustained speed.
Barili, A., Ceresa, M., \& Parisi, C. (1995). Energy-saving motion control for an autonomous mobile robot. In Industrial Electronics, 1995. ISIE '95, Proceedings of the IEEE International Symposium (vol. 2, pp. 674-676).

Bekker, M. G. (1969). Introduction to terrain-vehicle systems. Ann Arbor, MI: University of Michigan Press.

Berenz, V., Tanaka, F., \& Suzuki, K. (2012). Autonomous battery management for mobile robots based on risk and gain assessment. Artificial Intelligence Review, 37(3), 217-237.

Boice, K., Leo, A., Lee, J., Paulson, Jr., J., Skalny, M., \& Valascho, T. (2010). Baseline field testing of bb-2590 lithium-ion batteries using an iRobot FasTac 510 robot. Technical report, TARDEC.

Broderick, J., Tilbury, D., \& Atkins, E. (2012). Energy usage for UGVs executing coverage tasks. Proc. SPIE 8387.

Broderick, J. A., Tilbury, D. M., \& Atkins, E. M. (2014). Optimal coverage trajectories for a UGV with tradeoffs for energy and time. Autonomous Robots, 36(3), 257-271.

Campanari, S., Manzolini, G., \& de la Iglesia, F. G. (2009). Energy analysis of electric vehicles using batteries or fuel cells through well-to-wheel driving cycle simulations. Journal of Power Sources, 186(2), 464-477.

Ceraolo, M., \& Pede, G. (2001). Techniques for estimating the residual range of an electric vehicle. IEEE Transactions on Vehicular Technology, 50(1), 109-115.

Collins, S., Ruina, A., Tedrake, R., \& Wisse, M. (2005). Efficient bipedal robots based on passive-dynamic walkers. Science, 307(5712), 1082-1085.

Crenshaw, B. (1971). Soil/wheel interaction at high speed. Society of Automotive Engineers.

El-Shawarby, I., Ahn, K., \& Rakha, H. (2005). Comparative field evaluation of vehicle cruise speed and acceleration level impacts on hot stabilized emissions. Transportation Research Part D: Transport and Environment, 10(1), 13-30.

Grahn, M. (1991). Prediction of sinkage and rolling resistance for off-the-road vehicles considering penetration velocity. Journal of Terramechanics, 28(4), 339-347.

Guo, T., \& Peng, H. (2013). A simplified skid-steering model for torque and power analysis of tracked small unmanned ground vehicles. In American Control Conference (ACC), (pp. 1106-1111).

Jacoff, A., Sheh, R., Virts, A.-M., Kimura, T., Pellenz, J., Schwertfeger, S., \& Suthakorn, J. (2012). Using competitions to advance the development of standard test methods for response robots. In Proceedings of the Workshop on Performance Metrics for Intelligent Systems, PerMIS '12 (pp. 182-189), ACM: New York.

Kanayama, Y., Kimura, Y., Miyazaki, F., \& Noguchi, T. (1990). A stable tracking control method for an autonomous mobile robot. In Proceedings of IEEE International Conference on Robotics and Automation (pp. 384-389).

Lin, X., Perez, H. E., Siegel, J. B., Stefanopoulou, A. G., Li, Y., Anderson, R. D., Ding, Y., \& Castanier, M. P. (2013). On-line parameterization of lumped thermal dynamics in cylindrical lithium ion batteries for core temperature 
estimation and health monitoring. IEEE Transactions on Control Systems Technology, 21, 1745-1755.

Logan, D. G., Pentzer, J., Brennan, S. N., \& Reichard, K. (2012). Comparing batteries to generators as power sources for use with mobile robotics. Journal of Power Sources, 212(0), 130-138.

Mei, Y., Lu, Y.-H., Hu, Y., \& Lee, C. (2004). Energy-efficient motion planning for mobile robots. In Robotics and Automation, 2004. Proceedings, ICRA '04. 2004 IEEE International Conference (vol. 5, pp. 4344-4349).

Mei, Y., Lu, Y.-H., Hu, Y., \& Lee, C. (2005). A case study of a mobile robot's energy consumption and conservation techniques. In Advanced Robotics, 2005. ICAR '05. Proceedings, 12th International Conference (pp. 492-497).

Microrobotics, Inc. (2011). MIDG IIC specifications, accessed March 23, 2012.

Morales, J., Martinez, J., Mandow, A., Garcia-Cerezo, A., Gomez-Gabriel, J., \& Pedraza, S. (2006). Power analysis for a skid-steered tracked mobile robot. In Mechatronics, 2006 IEEE International Conference (pp. 420-425).

Morales, J., Martinez, J., Mandow, A., Garcia-Cerezo, A., \& Pedraza, S. (2009). Power consumption modeling of skidsteer tracked mobile robots on rigid terrain. IEEE Transactions on Robotics, 25(5), 1098-1108.

Ooi, C., \& Schindelhauer, C. (2009). Minimal energy path planning for wireless robots. Mobile Networks and Applications, 14, 309-321. doi: 10.1007/s11036-008-0150-5.

Pope, R. (1971). The effect of wheel speed on rolling resistance. Journal of Terramechanics, 8(1), 51-58.

Rakha, H. A., Ahn, K., Moran, K., Saerens, B., \& den Bulck, E. V. (2011). Virginia Tech comprehensive power-based fuel consumption model: Model development and testing. Transportation Research Part D: Transport and Environment, 16(7), 492-503.
Richmond, P. W., Mason, G. L., Coutermarsh, B. A., Pusey, J., \& Moore, V. D. (2009). Mobility performance algorithms for small unmanned ground vehicles. Technical report, DTIC Document.

Sadrpour, A., Jin, J., \& Ulsoy, A. (2012). Mission energy prediction for unmanned ground vehicles. In Robotics and Automation (ICRA), 2012 IEEE International Conference (pp. 2229-2234).

SAE International (2012). Electric vehicle energy consumption and range test procedure. Standard J1634.

Saha, B., Goebel, K., Poll, S., \& Christophersen, J. (2007). An integrated approach to battery health monitoring using Bayesian regression and state estimation. In Autotestcon, 2007 IEEE (pp. 646-653).

Say, M. G., \& Taylor, E. O. (1986). Direct current machines, 2nd ed. London: Pitman.

Shmulevich, I., Mussel, U., \& Wolf, D. (1998). The effect of velocity on rigid wheel performance. Journal of Terramechanics, 35(3), 189-207.

Tikhonov, A. N., \& Arsenin, V. Y. (1977). Solutions of ill-posed problems. Washington, DC: Winston.

Wang, H., Fu, L., Zhou, Y., \& Li, H. (2008). Modelling of the fuel consumption for passenger cars regarding driving characteristics. Transportation Research Part D: Transport and Environment, 13(7), 479-482.

Wong, J. (2008). Theory of ground vehicles. Wiley.

Wong, J., \& Chiang, C. (2001). A general theory for skid steering of tracked vehicles on firm ground. Proceedings of the Institution of Mechanical Engineers, Part D: Journal of Automobile Engineering, 215(3), 343-355.

Zhang, Y., Wang, W., Kobayashi, Y., \& Shirai, K. (2012). Remaining driving range estimation of electric vehicle. In Electric Vehicle Conference (IEVC), 2012 IEEE International (pp. $1-7)$. 